\title{
Natures Buildings as Trees: Biologically Inspired Glass as an Energy System
}

\author{
Mark Edward Alston \\ University of Salford Manchester, Manchester, UK \\ Email: M.E.Alston@salford.ac.uk
}

Received 13 March 2015; accepted 12 April 2015; published 15 April 2015

Copyright (C) 2015 by authors and Scientific Research Publishing Inc.

This work is licensed under the Creative Commons Attribution International License (CC BY). http://creativecommons.org/licenses/by/4.0/

(c) (i) Open Access

\section{Abstract}

The adaptive capacity in creating intelligent glass surfaces will be investigated using the principles of solar absorbance and active fluidic conductivity management as an energy system. To act as a thermal adsorption layer by applying bio-logically inspired engineering aims, of capture in enabling thermal transfer and control to regulate material composition. The creation of an adaptive cooling layer, by responsive measures to mirror our eco-systems through the employment of programmable self-awareness measures to regulate solar adsorption. These strategies for adaptation could enable the transformation of tall buildings, from mere material entities to mimic the intelligent surfaces of trees. Nature's eco-systems are living multi-functional mechanical information systems of chemical composition forming hierarchical structures. They have the ability to learn and adapt to changing climatic circumstance by self-regulation of solar adsorption, to achieve material thermal management. These programmable controls of adaptive material performance change in relationship to solar capture. Could this be harnessed to exploit the functionalities and behavior of materials on the surfaces of buildings to act as an energy system, by the application of bio-logically inspired engineering aims: 1) Material absorbency: thermal conductivity adsorption of solar irradiance. 2) Adaptive real-time performance: material autonomy.

\section{Keywords}

Energy, Biological Inspired Engineering, Solar, Adaptive, City Resilience

\section{Introduction}

Cities across the globe are responsible for up to $70 \%$ of global carbon emissions and $75 \%$ of global energy consumption. By 2050 it is estimated that $70 \%$ of the world's population will live in cities [1]. This drive of urban colonization puts greater demands for primary energy consumption. Predictions indicate that primary energy 
will grow at a higher rate than population increase per capita value by $15.7 \%$ over the next 30 years [2]. Today $80 \%$ of the world's primary energy comes from polluting high-carbon fossil fuels coal, oil and natural gas, IEA [3]. Cities and urban areas, consuming up to $80 \%$ of this generated energy, are at the same time part of the problem and part of the solution. This consumption of energy use by population in cities enabled measures for energy efficiencies to control energy demand [4]. These demands are driven by cities core temperature rise by the influence of annual solar radiation (approximately 3,400,000 EJ) [5] reaching the earth. This represents 7500 times the world's total annual energy consumption of 450 EJ. This is in order of magnitude greater than all the estimated non-renewable resources, including fossil fuels and nuclear. The construction and life-cycle habitation of buildings in the EU "would influence $42 \%$ of our final energy consumption, about $35 \%$ of our greenhouse gas emissions" [6]. COM 571 discusses the greater need for improved efficiencies in resources and energy use during the life cycle, in enabling resource efficient buildings. Studies by Earth Trends [7] indicate that buildings in Europe account on average for $36 \%$ of the energy use: the non-residential sector accounts for $8.7 \%$ and the residential sector for $27.5 \%$ of the total. The level of the energy consumption of a building depends on the building typology and geographical location. If energy consumption demand continues at its present rate and we continue to live in the same manner, we will need three planet earths to support and provide for our resources [8]. Energy consumption and the majority of carbon emissions release is associated with heating, moving air for cooling, water, and the use of appliances. To increase energy efficiency and reduce energy consumption requires a systemic approach for buildings and system technologies to improve life-cycle energy demands [9].

The development of multifunctional integrated building envelope systems, of high performance materials to enable thermal regulation management is the new frontier. This becomes increasingly significant for glass buildings where climatic temperatures are $>40$ degrees, as the demand for comfort conditions and quality of the indoor environments is affected by adsorbed solar heat.

Different kinds of technological solutions have been developed in response to improve energy-efficiency and sustainability for glass buildings. These include double facades, hybrid facades and decentralized mechanical services. Other facade technologies include external thermal insulating systems; ventilate facades, double skin glass facades; solar shading (lamellas or panels, replacing windows with Low-E high-performing glass); passive solar energy systems (solar glazing balconies); active solar systems (solar collectors, photovoltaic modules) [10]. Electrochromic windows have been used to regulate thermal radiation as the window can change its optical properties in response to an electric field and then returned to its original state by a field reversal. This switching is achieved by electrochromic crystalline tungsten oxide film of electrical conductivity ions to enable optical changes by the influence of an electric field [11] [12]. Transparent solid insulation material of light transmitting aerogel (comprising of $95 \%$ air) that inhibits heat transfer to enable solar transmittance has been discussed by Torgal [13]. The impact of these solutions is the reduction in luminosity as the light is scattered, hence the visible light entering the indoor space is reduced. Hence increased artificial lighting levels are required to compensate. Artificial Lighting is one of the biggest causes of energy-related greenhouse gas emissions. The total lighting-related $\mathrm{CO}_{2}$ emissions was estimated to be 1900 million tons [Mt] in 2005, which was about $7 \%$ of the total global $\mathrm{CO}_{2}$ emissions from the consumption and flaring of fossil fuels [14] [15]. According to US DoE figures [16], buildings may use between $15 \%$ - 35\% of their energy consumption for lighting.

Glass envelope performance is based upon measures in the reduction of heat conduction, to resolve the conflicts between services and fabric provisions (heating systems, fighting cooling systems). This response is a static one and does not reflect or adapt to it surrounding or understanding of geographical location. However eco systems have the ability to respond to the influence of ambient temperature, solar radiation gain, exposure to wind and changing micro climatic variation. These are adaptive strategies to influence and change material composition.

Nature's integrative functions to control conductivity would progress glass non-reactive envelopes. In response to thermal heat conductivity measures to reflect and sync with different regions to the context of buildings typology? To advance building fenestration to act as an adaptive cooling mechanism of high emissivity to regulate and give increased thermal conductivity management, as a bioengineering behavior approach? This adaptive multifunctional system of self-regulated conductivity behavior would enable energy production, distribution and storage technologies, integrated into a glass envelope. New methodologies are required to integrate comfort systems, energy management systems and local energy generation. This systemic approach will give improved comfort and quality of the indoor environment, to give optimal use of geographical location.

The aim of this paper is to put forward measures to create bio-inspired solution for glass to prevent uncon- 
trolled heating of this material and the effects of heat conduction, to create a high emissivity façade. To enable adaptive strategies, to control solar irradiance by molecular understanding of conductivity behavior, by self programmable intelligent surfaces. This adaption-cooling layer of hierarchical control will be able to regulate conductivity, to enable real-time thermal management of surfaces of tall buildings, by the objectives of:

- Material absorbency - thermal conductivity adsorption of solar irradiance.

- Material Autonomy-thermal management of microfluidic conductivity.

\section{Bio-Logically Inspired Engineering}

Biological materials react and give adaptive strategies in response to external stimuli by environment condition influence. These organic materials are multifunctional systems as they are mechanically strong and resilient formed by chemical composition. They are information systems that can self adapt to changes in ambient conditions by chemical and mechanical signals [17]. This ability to learn and self organize material composition by biochemical mechanisms is the process of continued development of materials at a evolutionary perspective. These materials have emerged through hierarchical self-assembly from nano scale components to determine the composite structure. These living cells are the ultimate intelligent materials to give reactive response to the environment and understanding of geographical location.

Plants and trees are transformable structures as they have autonomous self healing, intelligent surfaces (leaves), and are adaptive to light and color. The structure of a tree is a direct response to solar orientation; Figure 1, as the shape and distribution of the branches defines the tree structure. This geometry orientation has developed in response for the maximization of day light capture.

Light capture (interception of the leaves for photosynthesis processes) of the tree canopy is defined by rule based geometry, canopy volume, total leaf area density and angular distribution of leaf surfaces [18]. Beer's law on light interception computations can determine these parameters. This approach to solar orientation and adsorption of light energy by biochemical processes, are responsive measures, a dynamic system. Could this approach of nature's adaptive functions, of biologically inspired intelligent materials enable progression of realtime, reactive materials that form the surfaces of glass buildings? To develop glass from being a mere material entity, to becoming a dynamic energy system to regulate its own thermal conductivity levels, by the hour, season and weather conditions. This regulation could be pre-programmed, self-programmable intelligence by the influence of mechanical and algorithmic controls in response to solar and climatic environment influence?

\section{Bioinspired Glass Material}

To develop a composite glass material for enabling optimization of a multi functional glass façade, to control solar irradiance by thermal behavior, exchange and management of conductivity by fluidics. To move current static glass facades, to be an adaption layer, that can regulate its own thermal conductivity levels in response to absorptivity by the principles of (Figure 2):

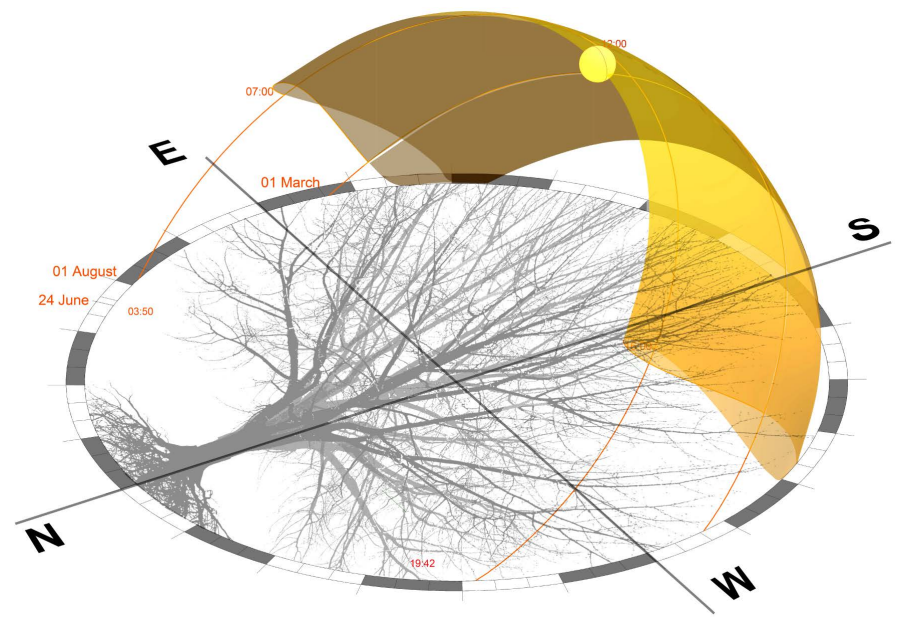

Figure 1. Trees structure geometry in relationship to solar orientation. 


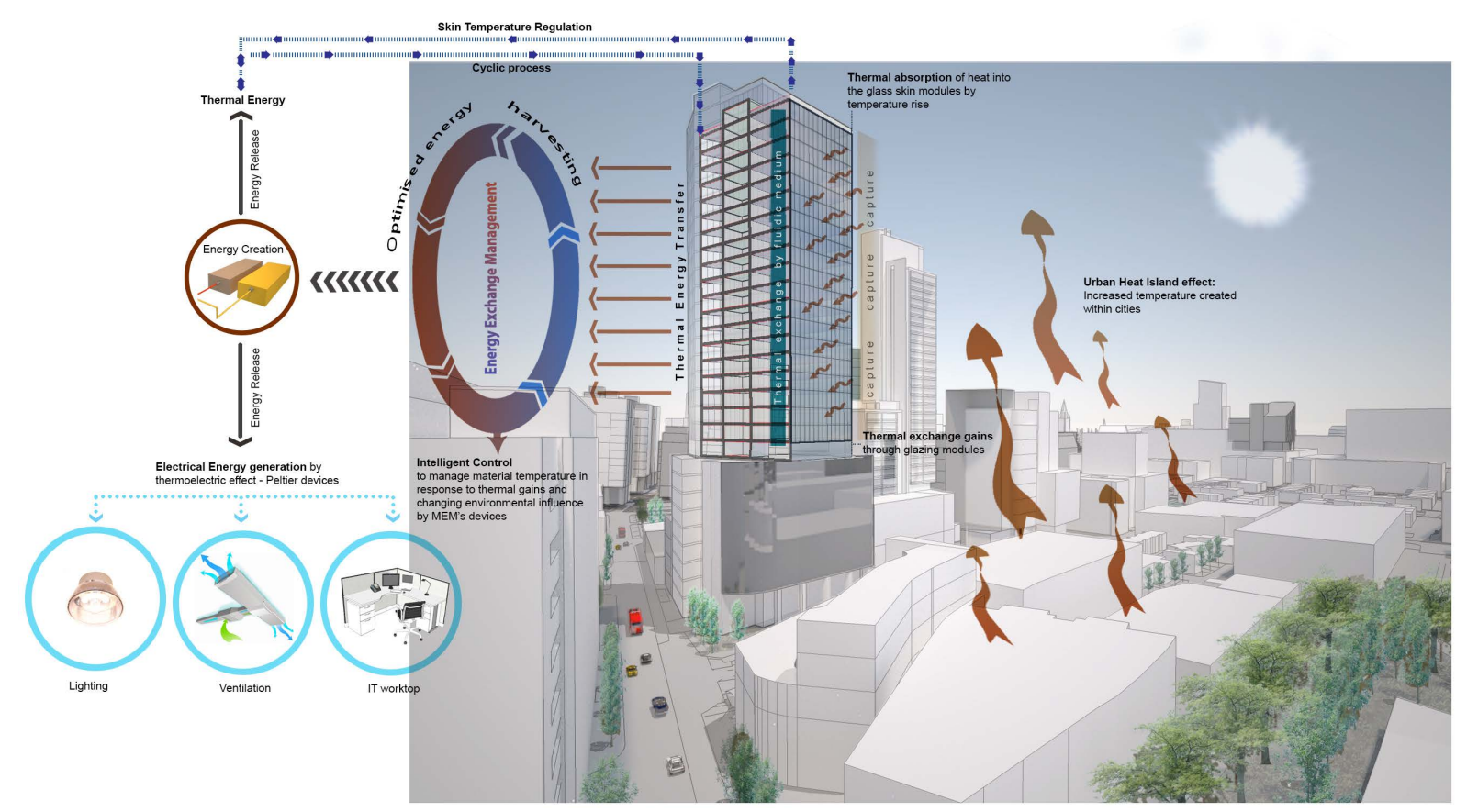

Figure 2. Abstract of glass adaptive response.

- Capture in enabling thermal transfer.

- Control to regulate and enable thermal management.

For conductivity control of glass, needs to be viewed as an energy system. To act as an absorber of thermal energy by molecular understanding of heat transfer, to enable thermal heat exchange between glass and another medium [fluidic] for thermal material management. Fluidics within vascular networks, capillary channels, are the methods employed by nature to regulate and control materials in response to solar irradiance. This absorptivity control will enable energy generation as a cyclic energy system [19] [20], is real time thermal management of material temperature regulation. The management of this energy flow would need to be supported by a decision support monitoring, to act and respond to fluctuations in material temperature. This thermal exchange and transfer by control of fluidic flow behavior, is a reactive system to external climatic temperature, Figure 3.

This flowchart model of sequential heat transfer is determined by setting steady state datum temperature. Any temperature rise is monitored by sensors and actuation systems that manipulate fluidic flows within a channel network, to react as a cyclic behavioral system for thermal management/regulation. The removal of adsorbed energy from the fluidic medium, Figure 3 (by thermal stores/electrical generation) enables cooling of the material to revert back to datum temperature. This is a cyclic action of molecule temperature regulation, is a repeated action for thermal energy adsorption. Rates of thermal adsorption are dependent upon the time of day, wind exposure, orientation and city temperature, heat island effects. This process to adsorb NIR, for stabilization of material temperature is the response to climatic influence and this is the approach of nature.

\section{Material Adsorbance}

Objective: thermal conductivity adsorption of solar irradiance.

Natures use fluidics' to act as a conductivity layer to adsorb direct transmitted solar radiation. Leaves for photosynthesis processes employ this principle. This method of using a fluidic medium as a means to adsorb inward thermal conduction and control radiation heat transfer is natures mechanisms to control conductivity. If shortwave irradiance/infrared spectrum NIR (but avoidance visible light) is captured and the energy obtained is removed, this process enables temperature control of the surrounding material. This control of thermal conductivity would enable cooling of a glass pane, reduction of glare and increase thermal comfort levels within building interiors. The ability to control solar irradiance depends on optical, thermal conductivity and the effects of geographical location. Research has been undertaken to introduce fluidics into windows. This research focused 


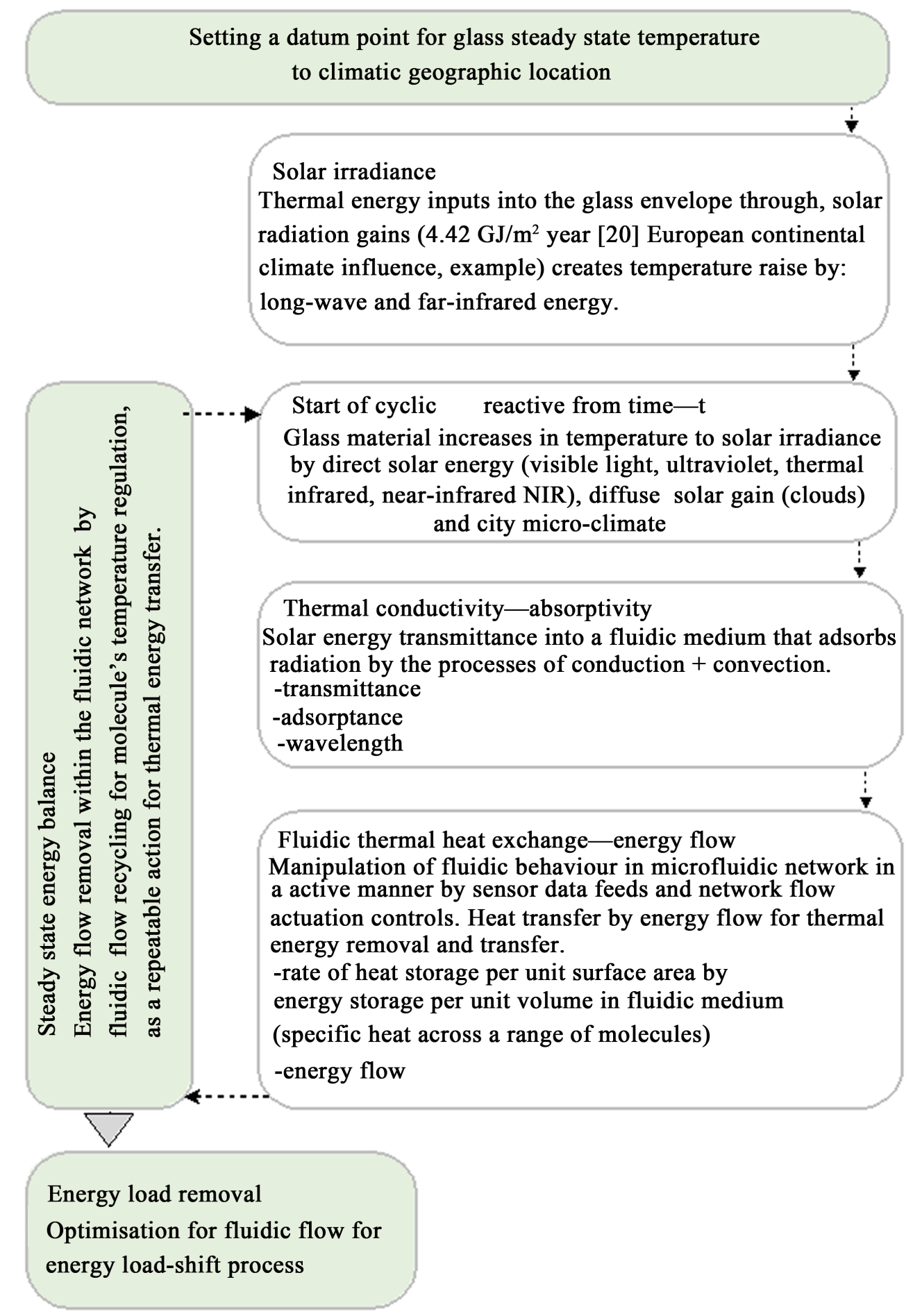

Figure 3. Simulation energy flowchart model.

on using the cavity between two planes of glass filled with water. This water flow gave cooling of glass surfaces by naturally flowed upward buoyancy and extraction of water for heating applications [21] [22]. The use of water enabled higher conductivity for effective window cooling designs in warm climates.

Results indicated adsorption of heat energy from solar gain was achieved, including indoor heat absorbency by thermal convection and radiation exchange giving a temperature difference of 10 degrees between inner glass 
plane. Water flow in the experiments was set at $200 \mathrm{ml} / \mathrm{min}$ with the greater efficiency gained in higher incident of solar radiation for working efficient conditions [23]. However as the angle of incidence fluctuates in relationship to solar radiation, effectiveness of the energy performance was diminished. This is due to free flowing, flow turbulence and, water under gravity flow within the cavity of the window that is uncontrolled. This created variations in temperature within the water volume between the two glass plane surfaces. The other critical issue was water flow velocity rates; this was considered to have great impact on the effectiveness to enable reduction for internal cooling load by fluid heat removal. The associated weight of a full volume, filled cavity of water dramatically added to the associated weight of the window unit, hence for a single window within a wall this could be contained. However applied to a fully glazed tall building the parameter have significantly changed, reducing the effectiveness of the application due to weight increase, management of flow turbulence and gravity flow. At this present time design of glass facades could be considered as one element, not withstanding this one element is comprised of a number of components. These components consist of and include frames, mullions, waterproofing gaskets, drainage channels, etc.

However could the glass envelope of a tall building be bracken down into autonomous self-regulating surfaces?

Each floor zone of a tall glazed building will have its own independent control in creating a cellular unit that will respond to conductivity. This cellular group would have active real time, autonomy for regulation/thermal management in connection to solar irradiance and effects. The benefit for this separation of one surface into multiple cellular groups to form the envelope, enables measures in response to solar orientation. These measures of real time reactive response are in relationship to changing wind patterns/exposure, adjacent building shadowing and micro-climatic influence within cities. This principle is similar to the layering of a leaf canopy in connection to leaf area density of a tree. Where each leaf and its relationship to geometry solar orientation is for the maximization of daylight capture.

Breaking down of one surface into horizontal cellular multi functional surfaces enable the movement of the fluidic medium working with gravity feed in avoidance of vertical upward transfer, for fluidic flow across consecutive floors in tall buildings. Hence fluidic flows within vascular networks can be contained to reduce pumping pressures and pumping measures in dealing with continuous tall glass surfaces of tall buildings, Figure 4.

\subsection{Vascular Network for Fluidic Regulation + Management}

The use of fluidics for thermal conductivity management of glass surfaces, enabling autonomy of thermal adsorption has advantages. Nature employs vascular networks to control temperature and heat transfer for heating or cooling as a behavior mechanism. This vasculature network acts as either a heat exchange layer for mammals vascularisation system or nutrient delivery layer for plants venation network for circulation of water, ions and photosynthate in a leaf. Microfluidic systems enable greater control of fluidic movement and optimization. These efficient capillary fluid networks control material temperature behavior by active fluidic flows. The insertion of this vascular network between two glazed planes would act as the adsorption layer for conductivity regulation. Microvascular volume filled fluidic networks, or matrixes, enables thermal exchange interactions to surround material regions, to actively effect its temperature as absorber of solar irradiance.

This application of continuously circulating a fluid into, through and out of the network, to adsorb and remove excess heat by active flow are bio-engineering principles. These volume filled 3D capillary networks are design to give optimization as analyzed by a multi objective algorithm [24] by the parameters of fluidic flow rate response, to achieve steady state temperature. Performance efficiency of the vascular networks geometry design, obtained by pre-programmed genetic algorithms [25]. This assessment of a network ability to adsorb thermal energy input by pre-programmable logic patterns, by the parameters of optimized flow efficiency enables manipulation of surface temperature. The network design is a balance of maintaining structural integrally of the medium/domain body in which the network is placed as a correlationship between flow performance requirements and network density patterns, for energy removal in response to conductivity. These networks of 3 dimensional geometries can be created in a number of differing mediums (PMMA, PDMS, epoxy matrix etc). Forming microvascular networks/capillaries can be constructed by varies means, CNC machines can fabricate by a layer by layer approach with network capillaries of tens of microns (feature sizes) by micro machining technology [26] [27]. This is network fabrication at a sub-micro resolution. The most innovative approach is the usage of ink writing to construct interpenetrating microvascular network, created by dual ink deposition using microcrystal 


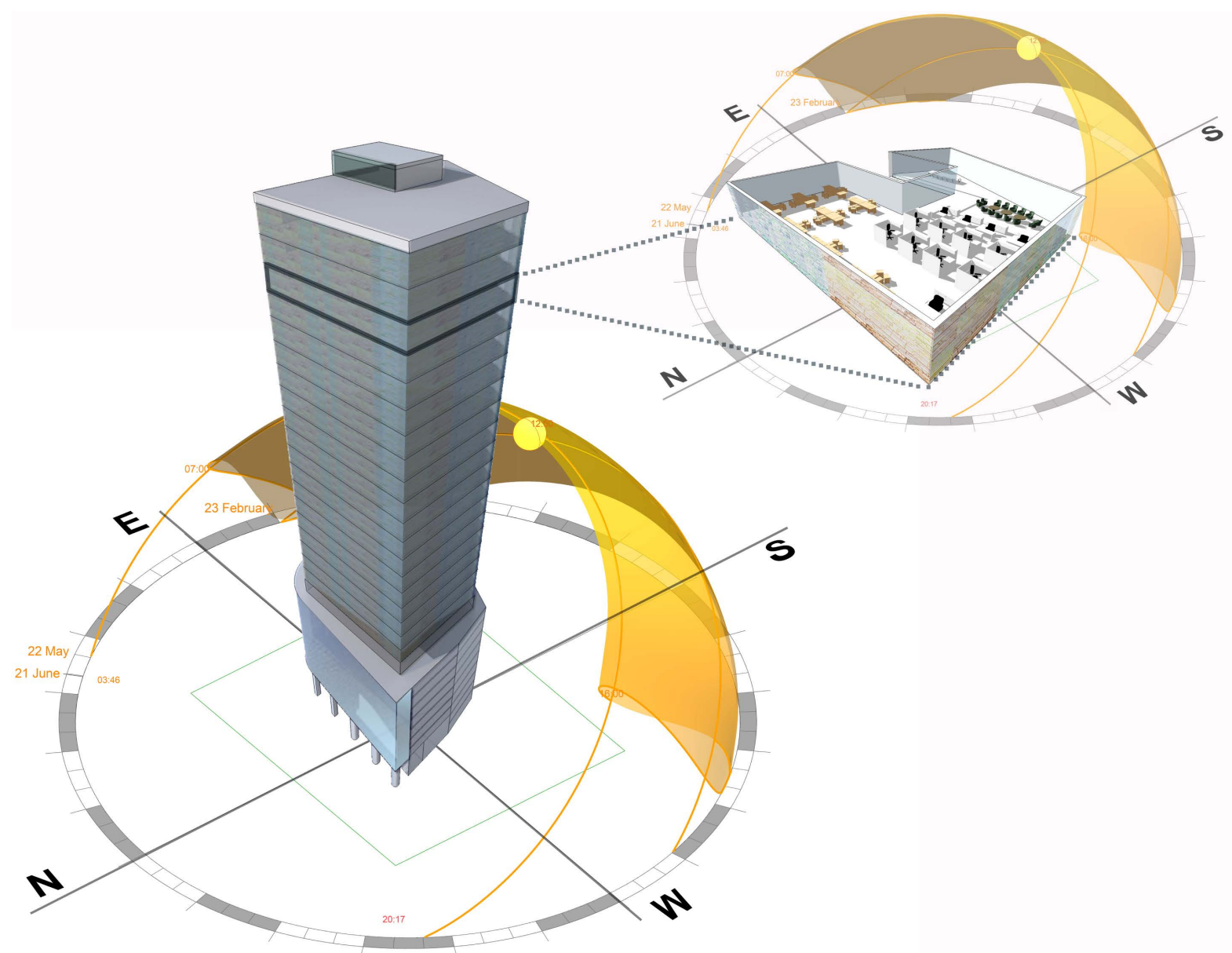

Figure 4. Cellular grouping to floor zone orientation.

line wax and mineral oils. This network is created by firstly printing the network in a 3D form and this is then encapsulated into a medium (epoxy/optically clear PDMS) with the removal of the wax based inks network by temperature liquefaction and vacuum. Removal of the inks creates a void within the domain material forming the vascular network, Figure 5.

An artificial network constructed in polydimethylsiloxane bonded to a single plane of glass with two differing capillary cross-sections $(1 \mathrm{~mm}$ by $0.10 \mathrm{~mm}$ and $2 \mathrm{~mm}$ by $0.10 \mathrm{~mm}$ ) has been tested by the Wyss Institute [28]. This PDMS material contained within its structure a series of capillary $100 \mu \mathrm{m}$ high channels created a diamond network patterns to enable fluidic water flow. A uniform heat load of 35 to 40 degrees was applied and measurements taken. Differing flow rates were introduced to measure the impacts of surface cooling of the glass plane. Variant flow rates assessed: $0.2 \mathrm{~mL} / \mathrm{min}, 2.0 \mathrm{~mL} / \mathrm{min}, 10 \mathrm{~m} / \mathrm{L} / \mathrm{min}$ were applied to the two prototype samples. The objective was to monitor the effectiveness of flow rates to achieve uniform steady state temperatures of the glass plane. Results concluded the effectiveness was dependent upon input water temperature to act as the absorber of thermal energy transfer, flow rate and material properties.

Figure 6 indicates significant temperature reduction was achieved at medium and high flow rates with water applied at 0 degree Celsius achieved dramatic response to mean temperature over time, with low flow rates being ineffective to control thermal temperature gains. The effectiveness of the vascular network was dependent on the capillary channel height/width, distances of the capillary channel junctions and the thickness of the medium, in this case PDMS. The results confirmed the effectiveness of the vascular network in actively enabling thermal adsorption and exchange. It was however the fluidic material itself that could have the greatest effect of convective heat transfer within the glass pane, this fluidic conductivity analysis was not explored in this experiment. 

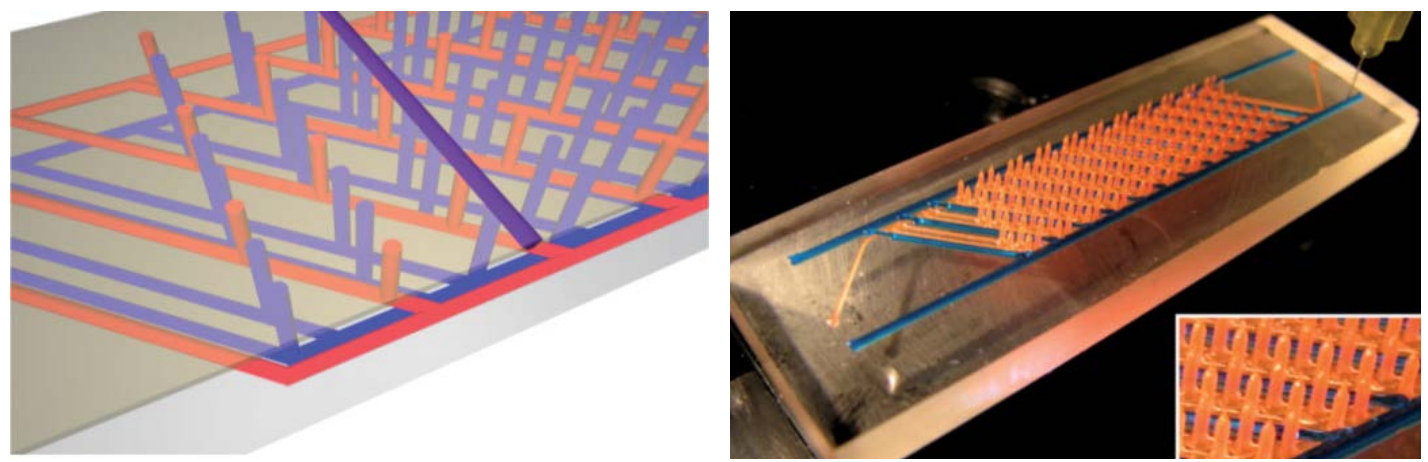

Figure 5. Vascular network created within a epoxy matrix-330 - $100 \mu \mathrm{m}$ capillaries [25].
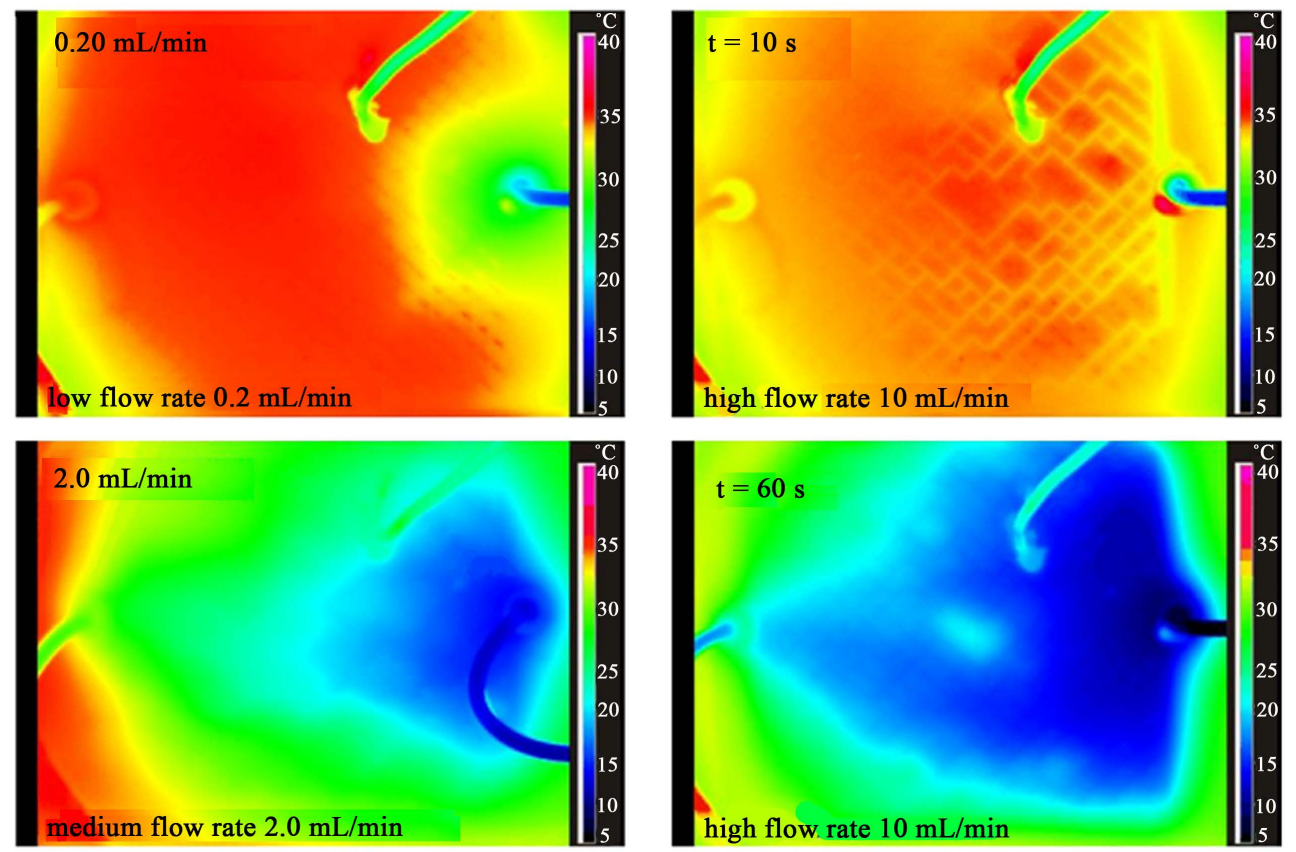

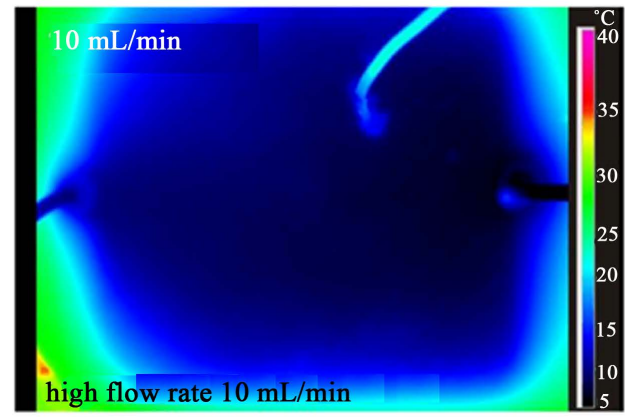

(a)

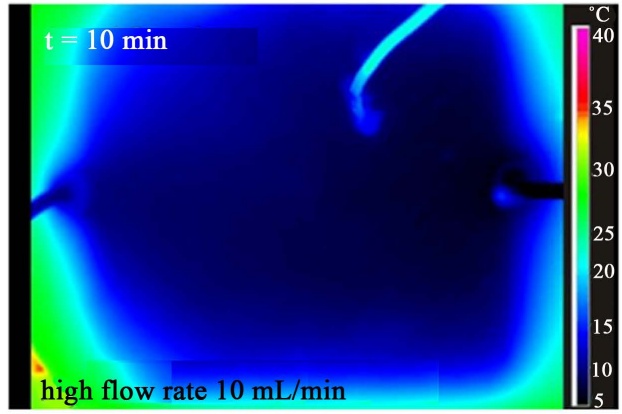

(b)

Figure 6. Influence of flow rates to achieve steady-state temperature of the two prototypes glass planes [28].

\subsection{Fluidic Thermal High, Energy Densities}

The understanding of fluidic energy densities to act as an optimized absorber is the solution objective to control material temperature in relationship to climate and orientation. To improve stored thermal energy within the fluidic medium, as a natural heat sink, significant amount of storage capacity is required within the transfer fluid, 
to manipulate material conductivity behavior. This absorbency of sensitive heat from air and solar radiation in keeping the glass pane at relatively low temperatures during the day is the objective. These are the same objectives nature employs to enable thermal management of material composition, human cardiovascular, plant vascular and mammals vasculature systems.

The performance of this fluidic medium as an optimal absorber must be in relationship to season conditions and retain high energy storage during the night compared to ambient temperature. This physically of thermal transfer from a heat carrier fluid to a storage system/electrical generation for energy down load process is a key cycle for active energy management. Until now the compromise between the thermo-physical characteristics, chemical stability and cost of material, the preferred selected fluidic medium is water. However this diffuse fluidic medium could be designed in regard to fluidic conductivity by molecular approach of synthesizing the material.

Nanofluidics in a vascular network would enable a designer fluidic approach to create thermal high, energy densities in connection to climate, orientation and microclimate variation. Nanofluidics are engineered colloids made of a base fluid and nanoparticles $(1-100 \mathrm{~nm})$. The presence of nanoparticles causes a dramatic enhancement of thermal conductivity [29], an increased of convective heat transfer coefficients as well as viscosity. These features makes nano fluids suitable for cooling and heat transportation applications by their heat transport properties:

- The suspended nanoparticles increase the surface area and the heat capacity of the fluid.

- The suspended nanoparticles increase the effective (or apparent) thermal conductivity of the fluid.

- The interaction and collision among particles, fluid and the flow passage surface are intensified as well as the mixing fluctuation and turbulence.

- The dispersion of nanoparticles fattens the transverse temperature gradient of the fluid.

With the development of nanotechnology, of improving thermal conductivity of heat transfer fluid, to suspend metal oxide nanoparticles or carbon nanotubes (smaller than $100 \mathrm{~nm}$ ) within it gives the evolution of fluidics synced to absorbency objective requirements.

The resulting mixture referred to as nanofluids possesses a substantially, larger thermal conductivity [30] compared to the original base fluids. Experimental studies conducted have shown that the effective thermal conductivity increases remarkably under macroscopically stationary conditions. This is also associated with enhancement of other transport properties (e.g., viscosity, specific heat, diffusivity, etc.). Hence, nanofluids are attractive materials for progressing and improving thermal conductivity of the heat transfer liquid. A starting point in accessing this fluidic material within the vascular network could be as simple as a water/salt hydrate solution and increasing its complexity to a chemical designer molecule approach. This chemical designer approach is the evolution of the fluidic medium absorbency optimization, to correlate to building (typology), geographic location and climatic influence set as a performance measure classification for our cities?

\section{Material Autonomy}

Objective: thermal management of microfluidic conductivity.

To control and influence material conductivity to enable self-regulation, mechanical flow rate control/information feeds are needed to actively manipulate fluidics within a microvascular network. The employment of hierarchical measures by sensors and actuators, controlled by programmable algorithms enables systems responses in real time. Consequently measurement of absorptivity is needed to give real-time response to changes and fluctuations in solar irradiance. The manipulation of fluidic adsorption by fluidic flows within a network, would create a cyclic behavioral system for thermal management/regulation, Figure 3, by the strategies of thermal energy load-unload processes. This load shift to remove energy from the fluidic medium to revert back to steady state temperatures enables thermal conductive management.

The entire glass fenestration to tall buildings could not be treated as one entity, as the vascular network will have a considerable resistance to flow. Pumping pressure needs to be controlled, as any energy gains from active conductivity control of glass would be outweighed by the pumping power requirement of the network. Hence the separation of the facade into cellular groups, Figure 4, to work with gravity and reduce pumping pressures is the start of this control process, Figure 7.

Cellular floor zoning creates unit floor cell autonomy by shared data communication interfaces and reactive changes of fluidic flow rates by actuators. This active management of flow rate cycle will feed into tank reservoirs 


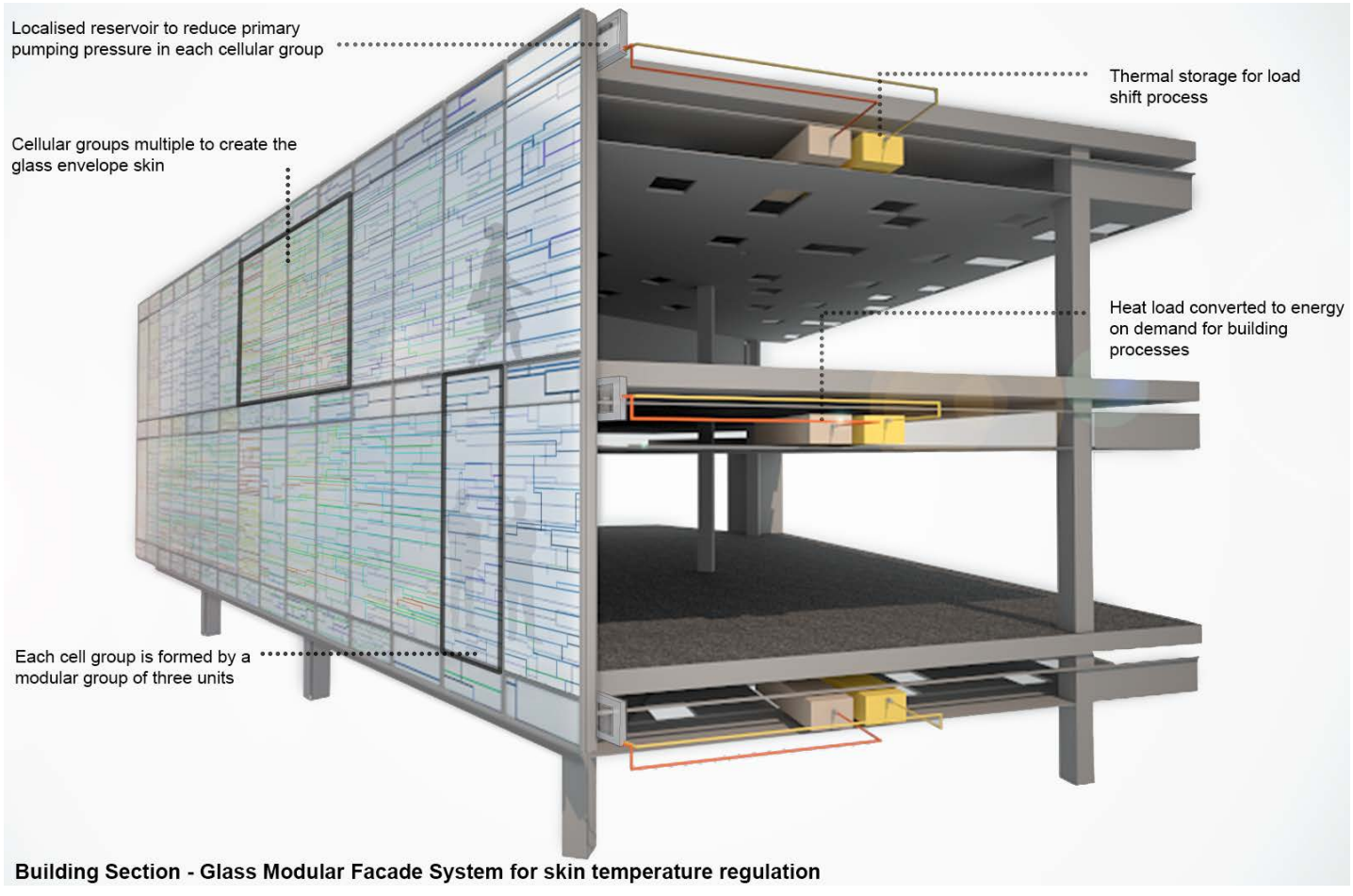

Figure 7. Cellular floor zoning-glass modular facade.

for energy removal from the vascular fluidic network enabling greater control of the module facade. This floor cell group will be able to learn and apply reactive response to changing irradiance and absorptivity. Energy unload process would be achieved by the creation of localized reservoirs, serving the cellular window groups to enable optimization of pumping power and energy load-shift processes, Figure 8. The ability to use tank storage reservoirs enables heat to electricity conversation by semiconducting engineering. The developments of semiconducting nanocomposites to yield high performance energy conversion [31] have been undertaken. The application of quantum dots, for thermopower enhancement of electrical conductivity for energy generation using thermopower, has strong application here. This coupled with thermostorage gives optimization capacity, in meeting consumer energy requirement demands per floor zone by creating of an autonomous energy community. The energy generation ratio demands for electrical/thermal could be synced to activity requirements per floor zone level (hotel, office, restaurant, health/spa, residential etc.) for multi functional typologies in tall buildings.

This optimization and syncing of energy generation to a specific activity and user energy demands, enables modeling energy profiling to each and every floor zone. The integration of multifunctional systems, including energy production, distribution and storage technologies, into a glass envelope, are new methodologies. The amalgamation of comfort systems for energy management and localized energy generation is the new technological frontier. The disconnection of the glass façade into, autonomous cellular zones, is a movement away from current technological solutions serve all approach. This objective connects energy demands to a multifunction glass envelope to reduce carbon emissions from reliance of one holistic approach to heating and cooling demands. The system functionality is however intrinsically linked to climatic influence and response. The selection, design and application of the fluidic medium within vascular networks, for thermal high densities optimization must be synced to geographical location. The optimization of integrated multifunctional objectives for glass facades for primary energy will enable energy performance and carbon footprint to sync with different regions and typology.

\section{Performance Response to Climate}

The energy consumer demands of a building depends on its typology, technological solutions, employed location and user specific issues, the most important of which are the following: 


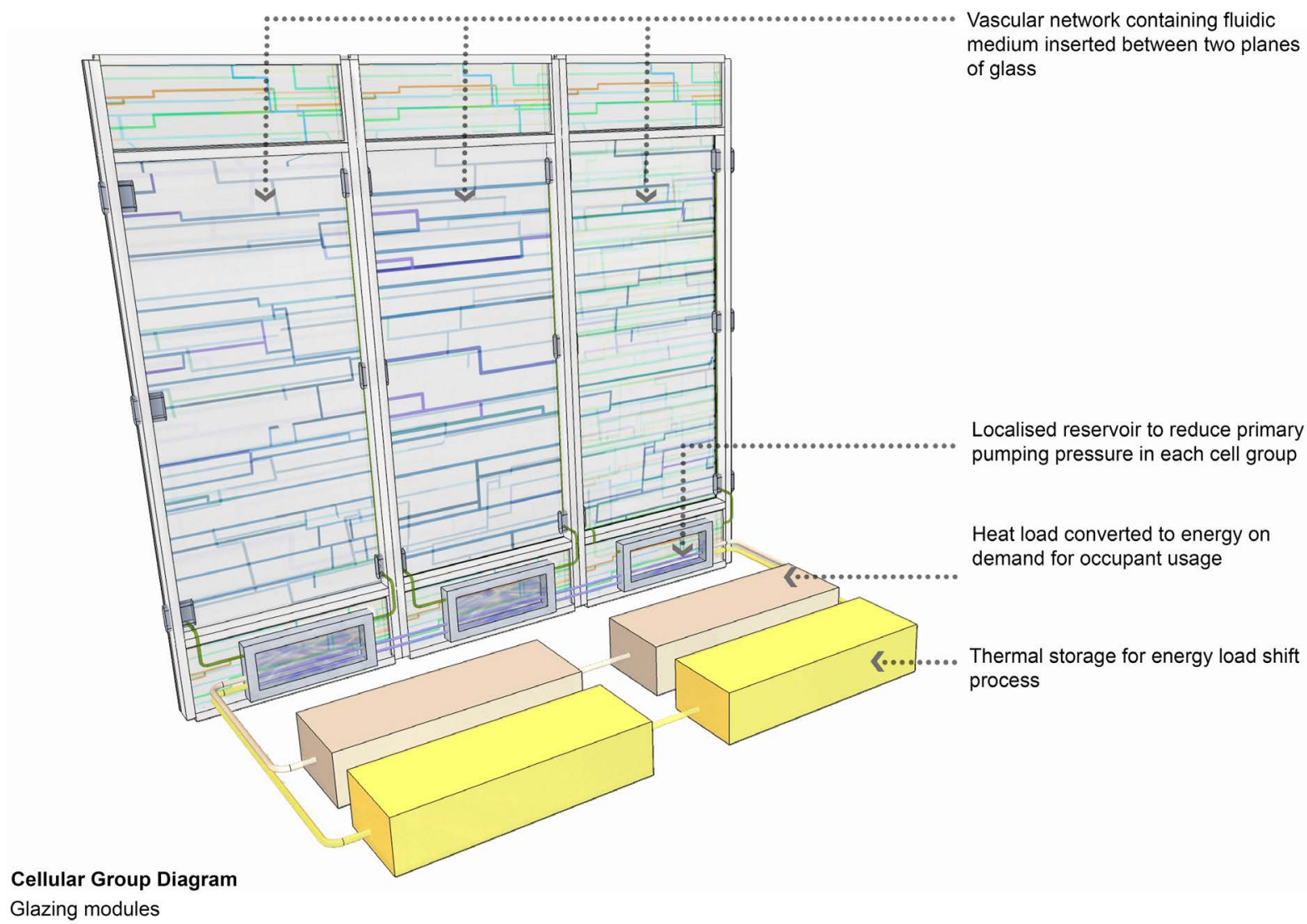

Figure 8. Modular cellular groups.

- Building typology in relationship to size, form, and placement on site.

- Climate, user occupant numbers, behaviour/required performance level.

- Insulation capacity of the envelope and thermal capacity.

- Efficiency of heating, ventilation, water supply and lighting systems, automation and control systems.

Energy efficiency enhancements would be achieved by applying new concepts of heating and/or cooling sources that can influence and reduce consumer energy consumption demands. Climate is having greater impacts on energy requirements by the increasing dependence to cool buildings in warm climates and this is indicated in the heating degree days (HDD). VTT project has evaluated the potentials on a European level to assess climatic effects to measure impacts for inherent measures to building facades. To measure technical solutions of facade behavior, to climatic conditions, in connection to building typologies was observed. Results indicated in Southern European countries, cooling demand becomes increasingly important for the overall energy consumption of a building due to higher requirements of thermal comfort [32]. The study found that in warm climatic zones the cooling demand can be reduced drastically by a combination of lowering the internal heat loads by greater control of thermal conductivity. This had environmental impacts upon the total primary energy demand according to climatic zone and building group/typology.

The development of adaptive glass facades as a optimal energy solution for different kinds of user energy demands, synced to a behavioral climatic model are new approaches. The setting of this climatic behavior model, Figure 3, will be depended upon the climatic classification in order to set primary data function in terms of steady state datum point. The understanding of the conductivity facade behavior of buildings and its implications on energy performance has been research through [32]. This research enabled a climatic classification analysis system to be developed.

Climatic categorizations are:

- Cfb-temperate without dry season, warm summer;

- Cfbw-temperate without dry season, warm summer and windy; 
- Csa-temperate with dry, hot summer;

- Dfb-cold, without dry season and with warm summer; and

- Dfc-cold, without dry season and with cold summer.

The design and selection of fluidic absorber in vascular networks of thermal high energy densities will have to be related to climatic categorization.

\section{Discussion}

Vascular, fluidic filled networks have the potential to change static buildings envelopes into reactive, dynamic, antonymous surfaces. In response to differing temperature ranges in relationship to solar gain and geographical location. The surfaces temperature of a building envelope has variation, due to orientation and city location. This imbalance in exposure to solar gain creates relatively cooler surfaces to north facing elevation, in comparison to others. Could this temperature differential be exploited?

The output thermal absorptivity of the fluidic material can be determined by the parameters below:

$$
\begin{gathered}
\mathrm{T}_{(x)}=\mathrm{T}_{\infty}+\left(\mathrm{T}_{\mathrm{O}}-\mathrm{T}_{\infty}\right) \exp \left(-\frac{\mathrm{A} \delta x}{\mathcal{Q}}\right) \\
\mathrm{T}_{\mathrm{IN}(x)}=\frac{\mathrm{T}_{x}+\mathrm{B}_{\rho} \mathrm{T}_{\text {room }}}{1+\mathrm{B}_{\rho}}
\end{gathered}
$$

$\mathrm{T}_{\infty}$ : Static water temperature

$\mathrm{B}_{\rho}: 0.13$ heart transfer Biot number-vascular domain material

$\mathrm{T}_{\mathrm{O}}$ : Inlet water temperature

$\mathrm{T}_{\text {room: }}$ : Room Temperature

A: Thermal coefficient of the domain material

$\mathrm{T}(x)$ : Temperature of cooling liquid in

$\mathrm{T}_{\mathrm{IN}(x)}$ : Glass surface internal temperature

$\delta x$ : capillary spacing of the vascular network

$\mathcal{Q}$ : fluidic flow rate

The output values of this absorptivity calculation will be specific to each building surface. The NIR input and conductivity output values will enable determination of the vascular network geometry design and response. Hence the functionality performance and complexity of the 3D network geometry could be linked to orientation. The absorbency and exposure of the fluidic material, Figure 9 would change in differing surfaces in response to solar orientation. This approach echo's nature's response, where leaf density patterns and leaf surface area, changes in response to solar gain.

The active NIR gain from southern surfaces could be moved to the relative cooler northern surfaces via the vascular network [33]. This would reduce the demands on energy unload processes, that could be considerable, of the fluidic absorber within the network by enabling reservoir tanks to be located on northern surfaces, per floor cellular zone. This movement of thermal energy from southern surfaces to relatively cooler northern surfaces, via fluidic thermal conductivity transfer, would have an impact on the capillary network geometry design. The capillary channels for fluidic flow would increase dimensionally, in order to meet the increased capacity flow requirements. This progression of increasing capillary channels dimensions enables greater volume flow rates and fluidic conductivity transfer for energy unloading, Figure 10.

Geometry design of the vascular network functionality response becomes linked to solar irradiance and orientation. Localized tank reservoirs placed to northern surfaces serving the cellular floor zone, Figure 4, enables storage and electrical energy generation to serve energy demands, with the ability to use changes in temperature, via orientation, to aid energy unload processes.

\section{Conclusion}

Our cities could be viewed as energy systems, using energy to supply its demands and creating heat island 


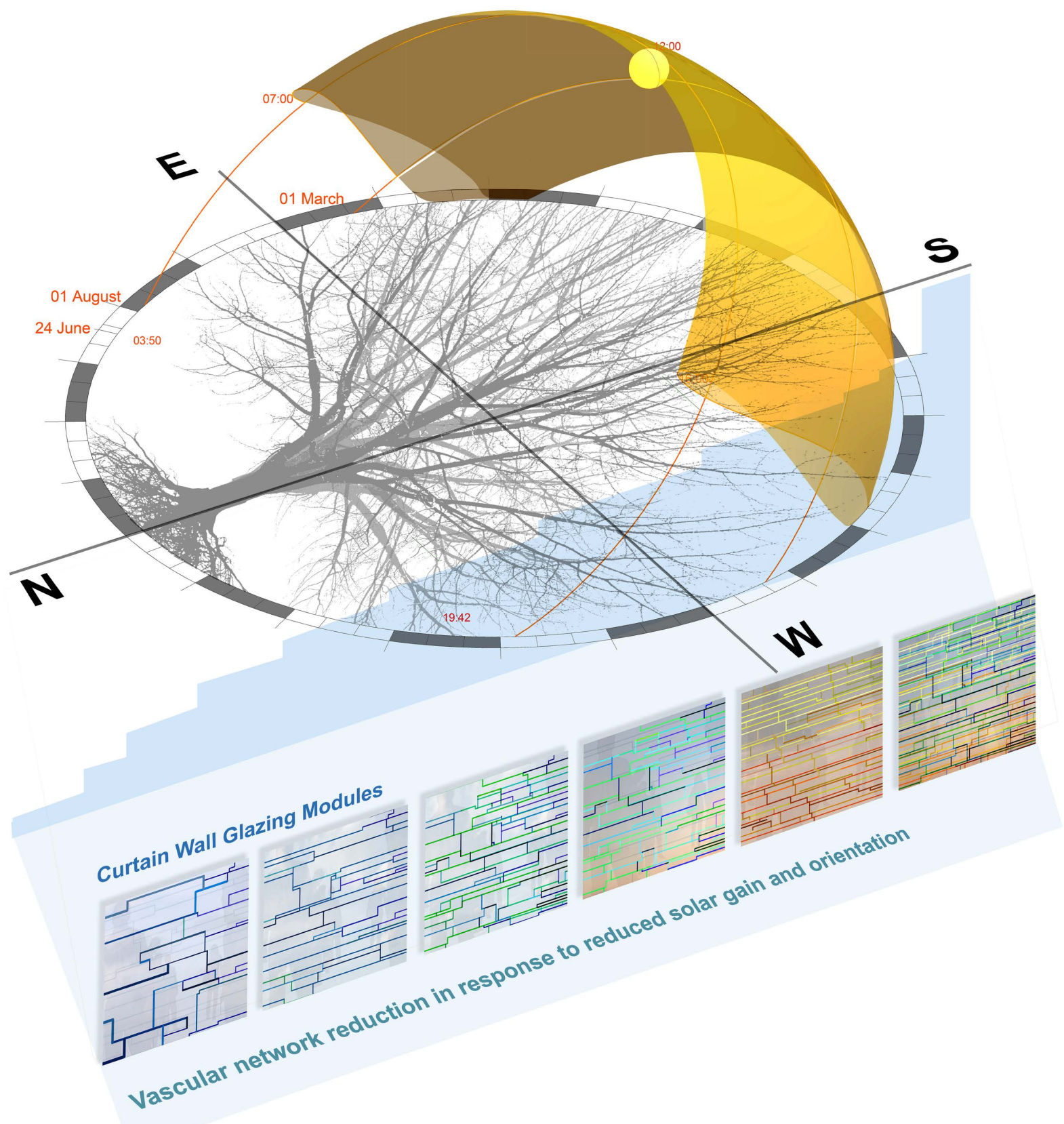

Figure 9. Vascular network geometry in response to solar orientation.

temperatures within the city core. To truly create pioneering smart cities, at the forefront of low carbon reduction, could we embrace new bio-inspired technological solutions? To change our facades into reactive dynamic systems by creating a adaptive thin cooling layer to control conductivity absorptivity by the hour, season and weather conditions. This systemic approach would improve comfort and quality of indoor environments by recognition of geographical location for the reduction of primary energy demands. This regulation and thermal conductivity management of the flow of energy or phases of energy creation is a balance in meeting consumer energy demand, as an energy network system. These principles to activity manage surface temperature of glass could change our buildings into climatic modifiers by the dehumidification of material temperature, that could create buildings working as clusters to influence city island temperatures. These factors of activity modification of temperature within cities could contribute to city resilience to reduce urban risk and measures to improve well 


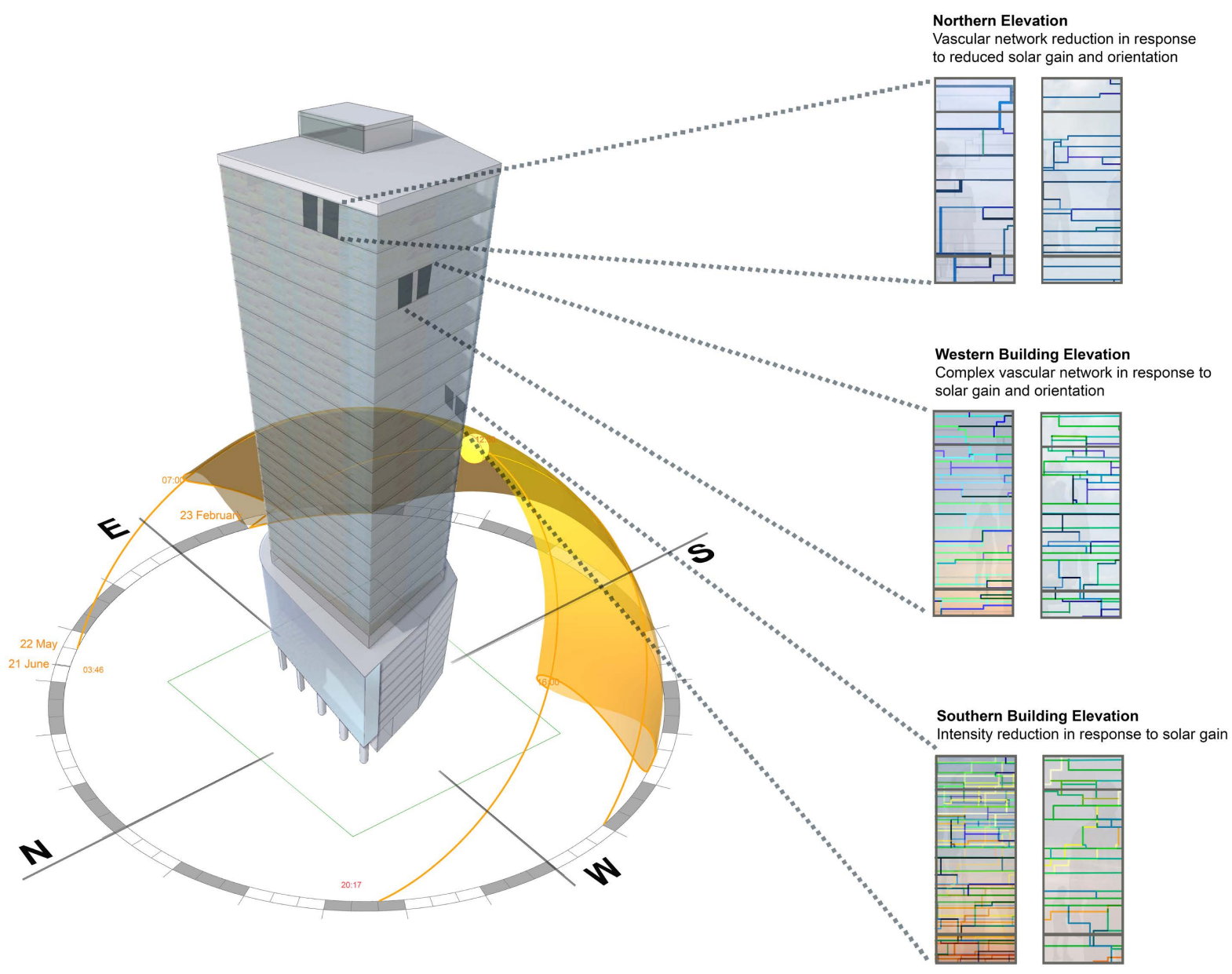

Figure 10. Vascular channel changes in response to solar orientation.

being of urban communities to create building resilience in an increasing unpredictable climatic world.

\section{Acknowledgements}

Dr Nikolaus Nestle, Research physicist at BASF SE, Ludwigshafen, Germany.

\section{References}

[1] Eames, M., Dixon, T., May, T. and Hunt, M. (2013) City Futures: Exploring Urban Retrofit and Sustainable Transitions. Building Research \& Information, 41, 504-516.

[2] Wackerernagel, M., Onisto, L., Bello, P., Callejas, L., Susana, L.F., Mendez, G. and Guadalupe, S.G. (1999) National Natural Capital Accounting with the Ecological Footprint Concept. Ecological Economics, 29, 375-390. http://dx.doi.org/10.1016/S0921-8009(98)90063-5

[3] Urban, F. (2009) Climate-Change Mitigation Revisited: Low-Carbon Energy Transitions for China and India. Development Policy Review, 27, 693-715. http://dx.doi.org/10.1111/j.1467-7679.2009.00466.x

[4] Brussels, COM 639 (2010) Communication from the Commission to the European Parliament, the Council, the European Economic and Social Committee and the Committee of the Regions. Energy 2020 A Strategy for Competitive, Sustainable and Secure Energy.

[5] Kumar, A., Kumar, K., Kaushik, N., Sharma, S. and Mishra, S. (2010) Renewable Energy in India: Current Status and Future Potentials. Renewable and Sustainable Energy Reviews, 14, 2434-2442. http://dx.doi.org/10.1016/j.rser.2010.04.003

[6] Brussels, COM 571 (211) Communication from the Commission to the European Parliament, the Council, the European Economic and Social Committee and the Committee of the Regions. Roadmap to a Resource Efficient Europe. 
[7] Earth Trends (2005) Terra Viva! Geo Data Guide.

[8] Kelly, M.J. (2009) Retrofitting the Existing UK Building Stock. Building Research \& Information, 37, 196-200. http://dx.doi.org/10.1080/09613210802645924

[9] Ramesh, T., Prakash, R. and Shukla, K. (2010) Life Cycle Energy Analysis of Buildings: An Overview. Energy and Buildings, 42, 1592-1600. http://dx.doi.org/10.1016/j.enbuild.2010.05.007

[10] Baeten, R., Jelle, B.P. and Gustavsen, A. (2010) Properties, Requirements and Possibilities of Smart Windows for Dynamic Daylight and Solar Energy Control in Buildings: A State-of-the-Art Review. Solar Energy Materials and Solar Cells, 94, 87-105. http://dx.doi.org/10.1016/j.solmat.2009.08.021

[11] Granqvist, C.G. (1995) Handbook of Inorganic Electrochromic Materials. Elsevier, Amsterdam.

[12] Kraft, A. and Rottmann, M. (2009) Properties, Performance and Current Status of the Laminated Electrochromic Glass of Gesimat. Solar Energy Materials \& Solar Cells, 93, 2088-2092. http://dx.doi.org/10.1016/j.solmat.2009.05.010

[13] Paceco-Torgal, F., Diamanti, M.V., Nazari, A. and Goran-Granqvist, C. (2013) Nanotechnology in Eco-Efficient Construction: Materials, Processes and Applications. Woodhead Publishing Series in Civil and Structural Engineering, Volume 43, Woodhead Publishing Ltd., Sawston.

[14] Annual Energy Outlook (2007) US Energy Information Administration, Washington DC.

[15] World Energy Outlook (2006) International Energy Agency, France.

[16] US Department of Energy (2006) Energy Demands on Water Resources.

[17] Chen, P.Y., Joanna, M.K. and Meyers, M.A. (2012) Biological Materials: Functional Adaptations and Bioinspired Designs. Progress in Materials Science, 57, 1492-1704. http://dx.doi.org/10.1016/j.pmatsci.2012.03.001

[18] Sinoquet, H., Sonohat, G., Phattaralerphong, J. and Godin, C. (2005) Foliage Randomness and Light Interception in 3D Digitised Trees: An Analysis from Multiscale Discretisation of the Canopy. Plant Cell and Environment, 28, 11581170.

[19] Alston, M.E. (2014) Energy Adaptive Glass Matter. Journal of Architectural Engineering Technology, 3, 115. http://dx.doi.org/10.4172/2168-9717.1000115

[20] Trees, Intelligent Energy-European Programme, Association pour la Rechercheet le Développement des Méthodes et ProcessusIndustriels. (2007).

[21] Chow, T.T., Chunying, L. and Zhang, L. (2011) Thermal Characteristics of Water-Flow Double-Pane Window. International Journal of Thermal Sciences, 50, 140-148. http://dx.doi.org/10.1016/j.ijthermalsci.2010.10.006

[22] Chow, T.T., Chunying, L. and Zhang, L. (2011) The Function of Solar Absorbing Window as Water-Heating Device. Building and Environment, 46, 955-960. http://dx.doi.org/10.1016/j.buildenv.2010.10.027

[23] Chow, T.T. and Chunying, L. (2013) Liquid-Filled Solar Glazing Design for Buoyant Water-Flow. Building and Environment, 60, 45-55. http://dx.doi.org/10.1016/j.buildenv.2012.11.010

[24] Olugebefola, S.C., Aragon, M.A., Hansen, C.J., Hamilton, A. and Wu, W. (2010) Polymer Microvascular Networks Composites. Journal of Composite Materials, 44, 2587-2603. http://dx.doi.org/10.1177/0021998310371537

[25] Hansen, C.J., Wu, W., Toohey, K.S., Sottos, N.R. and White, S.R. (2009) Self-Healing Materials with Interpenetrating Microvascular Networks. Advanced Materials, 21, 4143-4147. http://dx.doi.org/10.1002/adma.200900588

[26] Chen, S. (2008) Fabrication of High-Density Micro-Holes by Upward Batch Micro EDM. Journal of Micromechanics and Microengineering, 18, Article ID: 085002. http://dx.doi.org/10.1088/0960-1317/18/8/085002

[27] Saxena, L., Agrawal, A. and Joshi, S. (2009) Fabrication of Microfilters Using Excimer Laser Micromachining and Testing of Pressure Drop. Journal of Micromechanics and Microengineering, 19, Article ID: 025025.

[28] Hatton, B.D., Wheeldon, I., Hancock, M.J., Kolle, M. and Ingber, D.E. (2013) Artificial Vasculature for Adaptive Thermal Control of Windows. Solar Energy Materials and Solar Cells, 117, 429-436. http://dx.doi.org/10.1016/j.solmat.2013.06.027

[29] Liu, Y.D., Yue, Z., Ming, W.T. and Xiao, S.Z. (2009) Experimental Study of Thermal Conductivity and Phase Change Performance of Nanofluids PCMs. Journal of Microfluidics and Nanofluidics, 7, 579-584.

[30] Donghyun, S. and Debjyoti, B. (2010) Enhanced Thermal Properties of PCM Based Nanofluid for Solar Thermal Energy Storage. Proceedings of the ASME 2010 4th International Conference on Energy Sustainability, Phoenix, AZ, 17-22 May 2010.

[31] Liu, Y.F., Sahoo, P., Makongo, J.P.A., Zhou, X.Y., Kim, S.-J., Chi, H., Uher, C., Pan, X.Q. and Poudeu, P.F.P. (2013) Large Enhancements of Thermopower and Carrier Mobility in Quantum Dot Engineered Bulk Semiconductors. Journal of the American Chemical Society, 135, 7486-7495. http://dx.doi.org/10.1021/ja311059m

[32] VTT (2012) Energy Efficient Façade System for Building Retrofitting. FP7, ENV.

[33] Nestle, N. (2014) Research Scientist, BASF \& Junior Professor in Vorgriffsregelung: Personal Communication. 